\title{
On the autonomous metric on the group of area-preserving diffeomorphisms of the 2 -disc
}

\author{
MICHAEL BRANDENBURSKY \\ JAREK KĘDRA
}

Let $\boldsymbol{D}^{2}$ be the open unit disc in the Euclidean plane and let $\boldsymbol{G}:=\operatorname{Diff}\left(\boldsymbol{D}^{2}\right.$, area) be the group of smooth compactly supported area-preserving diffeomorphisms of $\boldsymbol{D}^{2}$. For every natural number $k$ we construct an injective homomorphism $\boldsymbol{Z}^{k} \rightarrow \boldsymbol{G}$, which is bi-Lipschitz with respect to the word metric on $\boldsymbol{Z}^{k}$ and the autonomous metric on $\boldsymbol{G}$. We also show that the space of homogeneous quasimorphisms vanishing on all autonomous diffeomorphisms in the above group is infinite-dimensional.

\section{Introduction}

\section{A The main result}

Let $\boldsymbol{D}^{2} \subset \boldsymbol{R}^{2}$ be the open unit disc and let $H: \boldsymbol{D}^{2} \rightarrow \boldsymbol{R}$ be a smooth compactly supported function. It defines a vector field

$$
X_{H}(x, y)=-\frac{\partial H}{\partial y} \partial_{x}+\frac{\partial H}{\partial x} \partial_{y}
$$

that is tangent to the level sets of $H$. Let $h$ be the time-one map of the flow $h_{t}$ generated by $X_{H}$. The diffeomorphism $h$ is area-preserving and every diffeomorphism arising in this way is called autonomous. Such a diffeomorphism is relatively easy to understand in terms of its generating function.

It is a well known fact that every smooth compactly supported and area-preserving diffeomorphism of the disc $\boldsymbol{D}^{2}$ is a composition of finitely many autonomous diffeomorphisms; see Banyaga [2]. How many? In the present paper we are interested in the geometry of this question. More precisely, we define the autonomous norm on the group $\boldsymbol{G}:=\operatorname{Diff}\left(\boldsymbol{D}^{2}\right.$, area) of smooth compactly supported area-preserving diffeomorphisms of the disc by

$$
\|f\|_{\text {Aut }}:=\min \left\{m \in N \mid f=h_{1} \cdots h_{m}, \text { where each } h_{i} \text { is autonomous }\right\} .
$$


The associated metric is defined by $\boldsymbol{d}_{\text {Aut }}(f, g):=\left\|f g^{-1}\right\|_{\text {Aut }}$. Since the set of autonomous diffeomorphisms is invariant under conjugation the autonomous metric is bi-invariant.

Theorem 1 For every natural number $k \in N$ there exists an injective homomorphism $\boldsymbol{Z}^{k} \rightarrow \operatorname{Diff}\left(\boldsymbol{D}^{2}\right.$, area) which is bi-Lipschitz with respect to the word metric on $\boldsymbol{Z}^{k}$ and the autonomous metric on $\operatorname{Diff}\left(\boldsymbol{D}^{2}\right.$, area).

\section{B Remarks}

(1) We show in the proof of Theorem 1 that the embedding of $Z^{k}$ is constructed to be in the kernel of the Calabi homomorphism $\mathcal{C}: \boldsymbol{G} \rightarrow \boldsymbol{R}$ (see Remark 2.2 for a definition and Banyaga [2], Calabi [10] and McDuff and Salamon [19] for more information).

(2) Gambaudo and Ghys defined in [13, Section 6.3] the autonomous metric on the group of area-preserving diffeomorphisms of the 2 -sphere and showed that its diameter is infinite.

(3) For each $p \in[1, \infty)$ the group $\boldsymbol{G}$ may be equipped with the right-invariant $L^{p}$ metric; see Arnol'd-Khesin [1] for a detailed exposition. Results similar to ours with respect to the $L^{p}$-metric were obtained by Benaim-Gambaudo in [3] and by the first named author in [7].

(4) In a greater generality, the autonomous metric is defined on the group $\operatorname{Ham}(M, \omega)$ of compactly supported Hamiltonian diffeomorphisms of a symplectic manifold. It would be interesting to know if such a metric is always unbounded.

(5) Even more generally the autonomous metric can be defined as follows. A compactly supported diffeomorphism $h$ of a manifold $M$ is called autonomous if it is the time-one map of a time-independent compactly supported flow $h_{t}$. The group $\operatorname{Diff}\left(\boldsymbol{D}^{2}\right)$ of all smooth compactly supported diffeomorphisms of the disc is generated by autonomous diffeomorphisms and hence the autonomous metric can be defined as above. However, it is known due to Burago, Ivanov and Polterovich [9], that the autonomous metric is bounded in this case.

(6) Since the autonomous metric is bi-invariant, investigating geometric properties of embeddings of nonabelian groups has to be done with respect to some bi-invariant metrics. At the time of writing this paper such metrics are not well understood. In Section 4 we prove an algebraic result which may indicate some good geometric properties. More precisely, for a nonabelian free group of rank two we construct an injective homomorphism $\boldsymbol{F}_{2} \rightarrow \boldsymbol{G}$ and prove that the image of the induced homomorphism $Q(\boldsymbol{G}) \rightarrow Q\left(\boldsymbol{F}_{2}\right)$ on the spaces of homogeneous quasimorphisms is infinite-dimensional. 


\section{C Comments on the proof of Theorem 1}

Let us start with a definition. A function $\psi: \Gamma \rightarrow \boldsymbol{R}$ from a group $\Gamma$ to the reals is called a quasimorphism if there exists a real number $A \geq 0$ such that

$$
|\psi(g h)-\psi(g)-\psi(h)| \leq A
$$

for all $g, h \in \Gamma$. The infimum of such numbers $A$ is called the defect of $\psi$ and is denoted by $D_{\psi}$. If $\psi\left(g^{n}\right)=n \psi(g)$ for all $n \in Z$ and all $g \in \Gamma$ then $\psi$ is called homogeneous. Any quasimorphism $\psi$ can be homogenized by setting

$$
\bar{\psi}(g):=\lim _{p \rightarrow+\infty} \frac{\psi\left(g^{p}\right)}{p} .
$$

The vector space of homogeneous quasimorphisms on $\Gamma$ is denoted by $Q(\Gamma)$. For more details about quasimorphisms, see eg Calegari [11].

The first part of the proof is to show that the space $Q(\boldsymbol{G}$, Aut $)$ of homogeneous quasimorphisms on $\operatorname{Diff}\left(\boldsymbol{D}^{2}\right.$, area) that are trivial on the set of autonomous diffeomorphisms is infinite-dimensional. This is done by constructing (for $n \geq 3$ ) an injective linear map

$$
\mathcal{G}_{n}: Q\left(\boldsymbol{B}_{n}, \boldsymbol{A}_{n}\right) \longrightarrow Q(\boldsymbol{G}, \text { Aut }),
$$

where $\boldsymbol{B}_{n}$ denotes the braid group on $n$-strings and $\boldsymbol{A}_{n} \subset \boldsymbol{B}_{n}$ is a certain abelian subgroup defined in Section 2.

Remark 1.1 Notice that the existence of a nontrivial homogeneous quasimorphism $\psi: \boldsymbol{G} \rightarrow \boldsymbol{R}$ that is trivial on Aut $\subset \boldsymbol{G}$ implies that the autonomous norm is unbounded. Indeed, for every $f \in G$ we have that $|\psi(f)|=\left|\psi\left(h_{1} \cdots h_{m}\right)\right| \leq m D_{\psi}$ and hence for every natural number $n$ we get $\left\|f^{n}\right\|_{\text {Aut }} \geq\left(|\psi(f)| / D_{\psi}\right) n>0$, provided $\psi(f) \neq 0$.

The map $\mathcal{G}_{n}$ is defined in Section 2 and is induced from the construction due to Gambaudo and Ghys [13]. The fact that the quasimorphism $\mathcal{G}_{n}(\varphi)$ is trivial on the set of autonomous diffeomorphisms provided $\varphi$ is trivial on $\boldsymbol{A}_{\boldsymbol{n}}$ is proved in Section 3. The latter proof consists of two steps. First, we show that $\mathcal{G}_{n}(\varphi)$ is trivial on autonomous diffeomorphisms generated by certain Morse-type functions (Theorem 3.2). Secondly, the set of Morse-type functions is dense in the set of all functions with respect to the $C^{1}$-topology, and

$$
\mathcal{G}_{n}(\varphi): \boldsymbol{G} \longrightarrow \boldsymbol{R}
$$

is a continuous function; see Theorem 3.4.

It is known that the space $Q\left(\boldsymbol{B}_{n}, \boldsymbol{A}_{n}\right)$ is infinite-dimensional (see Section 3C) and hence we obtain that $Q(\boldsymbol{G}$, Aut $)$ is infinite-dimensional. Let $\varphi_{i}: \boldsymbol{B}_{3} \rightarrow \boldsymbol{R}$ be homogeneous 
quasimorphisms comprising a set of $k$ linearly independent elements of $Q\left(\boldsymbol{B}_{3}\right)$. It follows that the map $\Phi: \boldsymbol{G} \rightarrow \boldsymbol{R}^{k}$ defined by

$$
\Phi(f)=\left(\mathcal{G}_{3}\left(\varphi_{1}\right)(f), \ldots, \mathcal{G}_{3}\left(\varphi_{k}\right)(f)\right),
$$

is Lipschitz and its image is quasi-isometric to the whole of $\boldsymbol{R}^{k}$.

The second part of the proof is a construction of a homomorphism $\boldsymbol{Z}^{k} \rightarrow \boldsymbol{G}$ with the required properties. It is in fact a section of the map $\Phi: \boldsymbol{G} \rightarrow \boldsymbol{R}^{k}$ mentioned above. It is defined by constructing $k$ diffeomorphisms $f_{j} \in \boldsymbol{G}$ with disjoint supports (hence commuting) such that $\mathcal{G}_{3}\left(\varphi_{i}\right)\left(f_{j}\right)=\delta_{i j}$, where $\delta_{i j}$ is the Kronecker delta.

Remark 1.2 The above map $\Phi$ is a quasimorphism, if one defines an $\boldsymbol{R}^{k}$ valued quasimorphism analogously to the real valued one using an $L^{p}$-norm. Observe that there exists a nontrivial homogeneous quasimorphism $\boldsymbol{F}_{2} \rightarrow \boldsymbol{R}^{k}$ on the free group of rank two with the image quasi-isometric to $\boldsymbol{R}^{k}$ for every $k \in \boldsymbol{N}$. This follows from the fact that $Q\left(\boldsymbol{F}_{2}\right)$ is infinite-dimensional. However, none of such quasimorphisms admits a homomorphic section over $\boldsymbol{Z}^{k}$ for $k \geq 2$.

\section{The Gambaudo-Ghys construction}

Let us recall a construction, due to Gambaudo and Ghys [13, Section 5.2], which produces a quasimorphism on $\boldsymbol{G}$ from a quasimorphism on the pure braid group $\boldsymbol{P}_{\boldsymbol{n}}$.

Let $g_{t} \in \boldsymbol{G}$ be an isotopy from the identity to $g \in \boldsymbol{G}$ and let $z \in \boldsymbol{D}^{2}$ be a basepoint. For $y \in \boldsymbol{D}^{2}$ we define a loop $\gamma_{y, z}:[0,1] \rightarrow \boldsymbol{D}^{2}$ by

$$
\gamma_{y, z}(t):= \begin{cases}(1-3 t) z+3 t y & \text { for } t \in\left[0, \frac{1}{3}\right], \\ g_{3 t-1}(y) & \text { for } t \in\left[\frac{1}{3}, \frac{2}{3}\right], \\ (3-3 t) g(y)+(3 t-2) z & \text { for } t \in\left[\frac{2}{3}, 1\right] .\end{cases}
$$

Let $\mathrm{X}_{n}\left(\boldsymbol{D}^{2}\right)$ be the configuration space of all ordered $n$-tuples of pairwise distinct points in the disc $D^{2}$. It's fundamental group $\pi_{1}\left(\mathrm{X}_{n}\left(D^{2}\right)\right)$ is identified with the pure braid group $\boldsymbol{P}_{n}$. Let $z=\left(z_{1}, \ldots, z_{n}\right)$ in $\mathrm{X}_{n}(\boldsymbol{D})$ be a base point. For almost every $x=\left(x_{1}, \ldots, x_{n}\right) \in \mathrm{X}_{n}\left(\boldsymbol{D}^{2}\right)$ the $n$-tuple of loops $\left(\gamma_{x_{1}, z_{1}}, \ldots, \gamma_{x_{n}, z_{n}}\right)$ is a based loop in the configuration space $\mathrm{X}_{n}\left(\boldsymbol{D}^{2}\right)$. Since the group $\boldsymbol{G}$ is contractible (see eg [22, Corollary 2.6]), the based homotopy class of this loop does not depend on the choice of the isotopy $g_{t}$. Let $\gamma(g, x) \in \boldsymbol{P}_{n}=\pi_{1}\left(\mathrm{X}_{n}\left(\boldsymbol{D}^{2}\right), z\right)$ be an element represented by this loop. 
Let $\varphi: \boldsymbol{P}_{n} \rightarrow \boldsymbol{R}$ be a homogeneous quasimorphism. Define the quasimorphism $\Phi_{n}: \boldsymbol{G} \rightarrow \boldsymbol{R}$ and its homogenization $\bar{\Phi}_{n}: \boldsymbol{G} \rightarrow \boldsymbol{R}$ by

$$
\Phi_{n}(g):=\int_{\mathrm{X}_{n}\left(\boldsymbol{D}^{2}\right)} \varphi(\gamma(g ; x)) d x \quad \text { and } \quad \bar{\Phi}_{n}(g):=\lim _{p \rightarrow+\infty} \frac{\Phi_{n}\left(g^{p}\right)}{p} .
$$

Remark 2.1 The assertion that both the above functions are well defined quasimorphisms is proved in [6, Lemma 4.1]. Using the family of signature quasimorphisms on $\boldsymbol{P}_{n}$ (one for each $n$ ), Gambaudo-Ghys showed that $\operatorname{dim}(Q(\boldsymbol{G}))=\infty$. This fact was also proved in [5].

Remark 2.2 The Calabi homomorphism $\mathcal{C}: \boldsymbol{G} \rightarrow \boldsymbol{R}$ may be defined as follows:

$$
\mathcal{C}(g)=\int_{0}^{1} \int_{D^{2}} H(x, t) d x d t,
$$

where $H(x, t)$ defines a flow whose time-one map is $g$, see eg [19, Lemma 10.27]. The group $\boldsymbol{P}_{2}$ is infinite cyclic, hence every homogeneous quasimorphism $\varphi_{2}: \boldsymbol{P}_{2} \rightarrow \boldsymbol{R}$ is a homomorphism. Since the kernel of the Calabi homomorphism $\mathcal{C}$ is a simple group [2], we have that $\bar{\Phi}_{2}(g)=C \cdot \mathcal{C}(g)$ for every $g \in \boldsymbol{G}$, where $C$ is a real constant independent of $g$. A proof of this equality which does not rely on the theorem of Banyaga can be found in [12].

The above construction defines a linear map $Q\left(\boldsymbol{P}_{n}\right) \rightarrow Q(\boldsymbol{G})$. Let

$$
\mathcal{G}_{n}: Q\left(\boldsymbol{B}_{n}\right) \longrightarrow Q(\boldsymbol{G})
$$

be its composition with the homomorphism $Q(\iota): Q\left(\boldsymbol{B}_{n}\right) \rightarrow Q\left(\boldsymbol{P}_{n}\right)$ induced by the inclusion $\iota: \boldsymbol{P}_{n} \rightarrow \boldsymbol{B}_{n}$. Let $\boldsymbol{A}_{n} \subset \boldsymbol{B}_{n}$ be an abelian group generated by braids $\eta_{i, n}$ shown in Figure 1. Recall that $Q\left(\boldsymbol{B}_{n}, \boldsymbol{A}_{n}\right)$ denotes the space of homogeneous quasimorphisms on $\boldsymbol{B}_{n}$ that are trivial on $\boldsymbol{A}_{n}$ and that $Q(\boldsymbol{G}$, Aut $)$ denotes the space of homogeneous quasimorphisms on $\boldsymbol{G}$ that are trivial on autonomous diffeomorphisms.

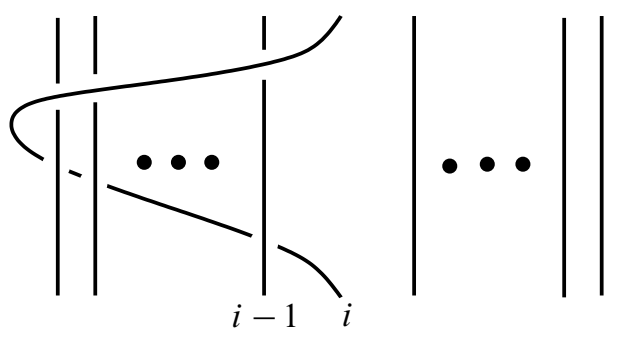

Figure 1: Braid $\eta_{i, n}$ 
Theorem 2.3 Let $n \geq 3$. The image of the linear map

$$
\mathcal{G}_{n}: Q\left(\boldsymbol{B}_{n}, \boldsymbol{A}_{n}\right) \longrightarrow Q(\boldsymbol{G}, \text { Aut })
$$

is infinite-dimensional. In particular, the diameter of $\left(\boldsymbol{G}, \boldsymbol{d}_{\mathrm{Aut}}\right)$ is infinite.

Remark 2.4 Theorem 2.3 answers the following question posed to the first author by L Polterovich:

Does there exist a quasimorphism on $\boldsymbol{G}$, given by the Gambaudo-Ghys construction, which vanishes on all autonomous diffeomorphisms in $\boldsymbol{G}$ ? In other words, does there exist a nontrivial element in $\operatorname{Im}\left(\mathcal{G}_{n}\right)$ which vanishes on all autonomous diffeomorphisms?

\section{Proofs}

\section{A Evaluation of the map $\mathcal{G}_{n}$ on autonomous diffeomorphisms}

Denote the space of autonomous compactly supported Hamiltonians $H: D^{2} \rightarrow \boldsymbol{R}$ by $\mathcal{H}$.

Definition 3.1 We say that a function $H \in \mathcal{H}$ is of Morse-type if:

(1) The boundary of the support of $H$ is a simple closed curve.

(2) The function $H$ has no degenerate critical points in the interior of its support.

(3) If $x, y$ are two distinct nondegenerate critical points of $H$ then $H(x) \neq H(y)$.

Theorem 3.2 If $\varphi_{n} \in Q\left(B_{n}, A_{n}\right)$ then $\bar{\Phi}_{n}(h)=0$ for every autonomous diffeomorphism $h$ generated by a Morse-type function $H$.

Proof The statement follows from [6, Theorem 4.5]. More precisely, it is shown there that

$$
\bar{\Phi}_{n}(h):=\mathcal{G}_{n}\left(\varphi_{n}\right)(h)=\int_{T} \hbar^{\prime} d \mu,
$$

where $T$ is the Reeb graph of $H, \hbar^{\prime}: T \rightarrow \boldsymbol{R}$ is a function induced by $H$ and $d \mu$ is a measure on $T$. All the objects above are defined in [6, Section 4.2]. In particular, it follows from the definition of the measure $d \mu$ that it is trivial if $\varphi_{n}\left(\eta_{i, n}\right)=0$ for each $i$. Hence $\bar{\Phi}_{n}(h)=0$, and the proof follows.

Remark 3.3 The idea of the proof relies on the fact that $n$ points on different level curves trace a braid conjugate to a braid in $\boldsymbol{A}_{n}$. 


\section{B The continuity of the Gambaudo-Ghys quasimorphisms}

The aim of this section is to prove the following result which will be used in the proof of Theorem 2.3.

Theorem 3.4 Let $H \in \mathcal{H}$ and $\left\{H_{k}\right\}_{k=1}^{\infty}$ be a sequence of functions such that each $H_{k} \in \mathcal{H}$ and $H_{k} \rightarrow H$ in $C^{1}$-topology. Let $h_{1}$ and $h_{1, k}$ be the time-one maps of the Hamiltonian flows generated by $H$ and $H_{k}$, respectively. Then

$$
\lim _{k \rightarrow \infty} \bar{\Phi}_{n}\left(h_{1, k}\right)=\bar{\Phi}_{n}\left(h_{1}\right) \text {. }
$$

The proof is presented below as a sequence of assertions and the theorem follows immediately from Proposition 3.8.

Let $g \in \boldsymbol{G}$ and $\left\{g_{t}\right\}_{t=0}^{1} \in \boldsymbol{G}$ such that $g_{0}=$ Id and $g_{1}=g$. We denote

$$
\mathfrak{G}\left(\left\{g_{t}\right\}\right):=\int_{\boldsymbol{D}^{2} \times \boldsymbol{D}^{2}} \frac{1}{2 \pi} \int_{0}^{1}\left\|\frac{\partial}{\partial t}\left(\frac{g_{t}(x)-g_{t}(y)}{\left\|g_{t}(x)-g_{t}(y)\right\|}\right)\right\| d t d x d y,
$$

where $\|\cdot\|$ is the Euclidean norm. $\mathfrak{G}\left(\left\{g_{t}\right\}\right)$ is well defined by [14, Lemma 1]. Denote

$$
\mathfrak{G}(g):=\inf _{g_{t}} \mathfrak{G}\left(\left\{g_{t}\right\}\right),
$$

where the infimum is taken over all isotopies $g_{t} \in \boldsymbol{G}$ joining the identity with $g$. By [14, Lemma 2] we have for all $g$ and $h$ in $\boldsymbol{G}$ that

$$
\mathfrak{G}(g h) \leq \mathfrak{G}(g)+\mathfrak{G}(h) .
$$

Thus the following limit exists:

$$
\mathfrak{L}(g)=\lim _{n \rightarrow \infty} \frac{\mathfrak{G}\left(g^{n}\right)}{n} .
$$

Proposition 3.5 Let $g \in \boldsymbol{G}$, and let $\varphi_{n}: \boldsymbol{B}_{n} \rightarrow \boldsymbol{R}$ be a homogeneous quasimorphism. Then

$$
\left|\bar{\Phi}_{n}(g)\right| \leq C_{1} \mathfrak{L}(g),
$$

where $C_{1}>0$ is independent of $g$.

Proof The proof of this proposition is very similar to the proof of [3, Lemma 4]. Let $x=\left(x_{1}, \ldots, x_{n}\right) \in X_{n}\left(\boldsymbol{D}^{2}\right)$ and $g_{t} \in \boldsymbol{G}$ joining the identity with $g$. Following [3, Section 4.1], to every $t \in[0,1]$ and $1 \leq i, j \leq n$ we associate the unit vector

$$
u\left(t, x_{i}, x_{j}\right)=\frac{g_{t}\left(x_{i}\right)-g_{t}\left(x_{j}\right)}{\left\|g_{t}\left(x_{i}\right)-g_{t}\left(x_{j}\right)\right\|} .
$$


Consider a map $u_{x_{i}, x_{j}}:[0,1] \rightarrow S^{1}$, where $t \rightarrow u\left(t, x_{i}, x_{j}\right)$. The change of variables induced by the map $u_{x_{i}, x_{j}}$ leads to the equality

$$
\mathfrak{G}\left(\left\{g_{t}\right\}\right)=\int_{\boldsymbol{D}^{2} \times \boldsymbol{D}^{2}} \frac{1}{2 \pi} \int_{\boldsymbol{S}^{1}} \#\left\{u_{x_{i}, x_{j}}^{-1}(\omega)\right\} d \omega d x_{i} d x_{j},
$$

where \# stands for cardinality. Consider the braid $\gamma(g ; x)$ defined by the isotopy $g_{t}$. Let $S \subset S^{1}$ be the set of all points $\omega$ in the circle for which the projection onto the line orthogonal to $\omega$ is injective on the set $\left\{x_{1}, \ldots, x_{n}\right\}$. The number of times the $i$-th strand overcrosses the $j$-th strand is bounded by \#\{ $\left\{u_{x_{i}, x_{j}}^{-1}(\omega)\right\}+4$, where $\omega \in S^{1} \backslash S$. The constant 4 comes from the fact that we close a path $g_{t}(x)$. Let $l(\gamma(g ; x))$ denote the length of the braid $\gamma(g ; x)$ with respect to the Artin generators $\left\{\sigma_{i}\right\}_{i=1}^{n-1}$ of $\boldsymbol{B}_{n}$. Note that the measure of the set $S$ is zero. It follows that for a generic $\omega \in \boldsymbol{S}^{1}$, ie for $\omega \in \boldsymbol{S}^{1} \backslash S$, we have

$$
l(\gamma(g ; x)) \leq \sum_{i \neq j}^{n}\left(\#\left\{u_{x_{i}, x_{j}}^{-1}(\omega)\right\}+4\right) ;
$$

see also the proof of [3, Lemma 4]. Consequently, we have that

$$
l(\gamma(g ; x)) \leq \sum_{i \neq j}^{n} \int_{S^{1}}\left(\#\left\{u_{x_{i}, x_{j}}^{-1}(\omega)\right\}+4\right) d \omega .
$$

Note that since $\varphi_{n}$ is a homogeneous quasimorphism there exists a positive constant $A$ such that $\left|\varphi_{n}(\alpha)\right| \leq A \cdot l(\alpha)$ for each $\alpha \in \boldsymbol{B}_{n}$. Let $u_{p, x_{i}, x_{j}}$ be the above function corresponding to the diffeomorphism $g^{p}$. It follows from (2) and (3) that

$$
\begin{aligned}
\left|\Phi_{n}(g)\right| & \leq A \lim _{p \rightarrow \infty} \int_{\mathrm{X}_{n}\left(\boldsymbol{D}^{2}\right)} \frac{l\left(\gamma\left(g^{p} ; x\right)\right)}{p} d x \\
& \leq A \lim _{p \rightarrow \infty} \int_{\mathrm{X}_{n}\left(\boldsymbol{D}^{2}\right)} \frac{\sum_{i \neq j}^{n} \int_{\boldsymbol{S}^{1}}\left(\#\left\{u_{p, x_{i}, x_{j}}^{-1}(\omega)\right\}+4\right) d \omega}{p} d x \\
& \leq(2 \pi)^{n-2} A \lim _{p \rightarrow \infty} \frac{1}{p} \sum_{i \neq j}^{n} \int_{\boldsymbol{D}^{2} \times \boldsymbol{D}^{2}} \int_{\boldsymbol{S}^{1}}\left(\#\left\{u_{p, x_{i}, x_{j}}^{-1}(\omega)\right\}+4\right) d \omega d x_{i} d x_{j} \\
& \leq(2 \pi)^{n-2} A \lim _{p \rightarrow \infty} \frac{1}{p} \sum_{i \neq j}^{n} 2 \pi \mathfrak{G}\left(\left\{g_{t, p}\right\}\right),
\end{aligned}
$$

where $g_{t, p}$ is any isotopy from the identity to $g^{p}$. Since the above inequalities hold for any isotopy between the identity and $g^{p}$ we have

$$
\left|\Phi_{n}(g)\right| \leq(2 \pi)^{n-1} n(n-1) A \lim _{p \rightarrow \infty} \frac{\mathfrak{G}\left(g^{p}\right)}{p} \leq(2 \pi)^{n-1} n(n-1) \mathfrak{L}(g)
$$

and the proof follows. 
Now we will recall a definition of the right-invariant $L^{2}$-metric on $\boldsymbol{G}$. It is defined as follows. Let

$$
L_{2}\left\{g_{t}\right\}:=\int_{0}^{1} d t\left(\int_{D^{2}}\left\|\dot{g_{t}}(x)\right\|^{2} d x\right)^{\frac{1}{2}}
$$

be the $L^{2}$-length of a smooth isotopy $\left\{g_{t}\right\}_{t \in[0,1]} \subset \boldsymbol{G}$, where $\left\|\dot{g}_{t}(x)\right\|$ denotes the Euclidean length of the tangent vector $\dot{g}_{t}(x) \in T_{x} D^{2}$. Observe that this length is right-invariant, that is, $L_{2}\left\{g_{t} \circ f\right\}=L_{2}\left\{g_{t}\right\}$ for any $f \in \boldsymbol{G}$. It defines a nondegenerate right-invariant metric on $\boldsymbol{G}$ by

$$
\boldsymbol{d}_{2}\left(g_{0}, g_{1}\right):=\inf _{g_{t}} L_{2}\left\{g_{t}\right\}
$$

where the infimum is taken over all paths from $g_{0}$ to $g_{1}$. See Arnol'd-Khesin [1] for a detailed discussion.

Corollary 3.6 Let $g \in \boldsymbol{G}$, and let $\varphi: \boldsymbol{B}_{n} \rightarrow \boldsymbol{R}$ be a homogeneous quasimorphism. Then

$$
\left|\bar{\Phi}_{n}(g)\right| \leq C_{3} d_{2}(\mathrm{Id}, g),
$$

where $C_{3}>0$ does not depend on $g$.

Proof It follows from [14, Theorem 1] that for any $g \in \boldsymbol{G}$ there exists a universal constant $C>0$, such that $\mathfrak{L}(g) \leq C \boldsymbol{d}_{2}(\mathrm{Id}, g)$. Now take $C_{3}=C \cdot C_{1}$, then the statement follows from Proposition 3.5.

Lemma 3.7 Let $F \in \mathcal{H}$. Then for any $\varepsilon>0$ and $p \in N$ there exists $\delta_{p}>0$, such that if $H \in \mathcal{H}$ is $\delta_{p}$-close to $F$ in $C^{1}$-topology, then

$$
\boldsymbol{d}_{2}\left(f_{1}^{p}, h_{1}^{p}\right)<\varepsilon
$$

where $f_{t}$ and $h_{t}$ are the Hamiltonian flows generated by $F$ and $H$.

Proof For the convenience we normalize the area of $\boldsymbol{D}^{2}$ to be 1. It is enough to show that for all $p \in N$ there exists $\delta_{p}>0$ such that

$$
\max _{x \in \boldsymbol{D}^{2}}\left\|\nabla F_{(x)}-\nabla H_{(x)}\right\|<\delta_{p} \Rightarrow \boldsymbol{d}_{2}\left(f_{1}^{p}, h_{1}^{p}\right)<\frac{\varepsilon}{2} .
$$

Note that $\boldsymbol{d}_{2}\left(f_{1}^{p}, h_{1}^{p}\right)=\boldsymbol{d}_{2}\left(\mathrm{Id}, f_{1}^{p} h_{1}^{-p}\right) \leq L_{2}\left(\left\{f_{t}^{p} h_{t}^{-p}\right\}\right)$. It follows from [21, Proposition 1.4.D] that

$$
\frac{\partial\left(f_{t}^{p} h_{t}^{-p}\right)}{\partial t}(x)=p \cdot\left(X_{F}-X_{\left(H f_{t}^{-p}\right)}\right)_{\left(f_{t}^{p} h_{t}^{-p}(x)\right)} \cdot
$$


Thus

$$
\begin{aligned}
\left\|\frac{\partial\left(f_{t}^{p} h_{t}^{-p}\right)}{\partial t}(x)\right\| & =p \cdot\left\|\left(X_{F}-X_{\left(H f_{t}^{-p}\right)}\right)_{\left(f_{t}^{p} h_{t}^{-p}(x)\right)}\right\| \\
& =p \cdot\left\|\left(\nabla F-\nabla\left(H f_{t}^{-p}\right)\right)_{\left(f_{t}^{p} h_{t}^{-p}(x)\right)}\right\|,
\end{aligned}
$$

Note that $f_{t}$ is an autonomous Hamiltonian flow. Thus $F f_{t}(x)=F(x)$ for all $x \in \boldsymbol{D}^{2}$ and $t \in \boldsymbol{R}$. It follows that for all $x \in \boldsymbol{D}^{2}$ and all $p \in \boldsymbol{Z}$,

$$
\nabla F_{\left(h_{t}^{-p}(x)\right)}\left(D f_{t}^{-p}\right)_{\left(f_{t}^{p} h_{t}^{-p}(x)\right)}=\nabla F_{\left(f_{t}^{p} h_{t}^{-p}(x)\right)} .
$$

We get the following inequality:

$$
\begin{aligned}
\boldsymbol{d}_{2}\left(f_{1}^{p},\right. & \left.h_{1}^{p}\right) \\
& \leq p \int_{0}^{1}\left(\int_{D^{2}}\left\|\left(D f_{t}^{-p}\right)_{\left(f_{t}^{p} h_{t}^{-p}(x)\right)}\right\|_{M}^{2} \cdot\left\|\nabla F_{\left(h_{t}^{-p}(x)\right)}-\nabla H_{\left(h_{t}^{-p}(x)\right)}\right\|^{2} d x\right)^{\frac{1}{2}} d t,
\end{aligned}
$$

where $\|\cdot\|_{M}$ is a matrix norm. Denote

$$
\mathfrak{M}_{D f_{t}}:=\max _{x \in \boldsymbol{D}^{2}, t \in[0,1]}\left\|\left(D f_{t}^{-1}\right)_{(x)}\right\|_{M} \quad \text { and } \quad \delta_{p}:=\frac{\varepsilon}{2 p\left(\mathfrak{M}_{D f_{t}}\right)^{p}} .
$$

We get the following inequality:

$$
\boldsymbol{d}_{2}\left(f_{1}^{p}, h_{1}^{p}\right) \leq p\left(\mathfrak{M}_{D f_{t}}\right)^{p} \max _{x \in \boldsymbol{D}^{2}}\|\nabla F(x)-\nabla H(x)\| \leq \frac{\varepsilon}{2} .
$$

It follows that if $H$ is $\delta_{p}$-close to $F$ in $C^{1}$-topology, then $\boldsymbol{d}_{2}\left(f_{1}^{p}, h_{1}^{p}\right)<\varepsilon$.

Proposition 3.8 Let $F \in \mathcal{H}$. Then for any $\varepsilon>0$ there exists $\delta>0$, such that if $H \in \mathcal{H}$ is $\delta$-close to $F$ in $C^{1}$-topology, then:

$$
\left|\bar{\Phi}_{n}(f)-\bar{\Phi}_{n}(h)\right| \leq \varepsilon,
$$

where $f$ and $h$ are time-one maps of flows generated by $F$ and $H$.

Proof Fix some $\varepsilon>0$. Let $D_{\Phi_{n}}$ be the defect of the homogeneous quasimorphism $\bar{\Phi}_{n}: \boldsymbol{G} \rightarrow \boldsymbol{R}$, and let $C_{3}$ be the constant which was defined in Corollary 3.6. Take $p \in N$ such that $\left(D \bar{\Phi}_{n}+C_{3}\right) / p<\varepsilon$. It follows from Lemma 3.7 that there exists $\delta_{p}>0$, such that if $H$ is $\delta_{p}$-close to $F$ in $C^{1}$-topology, then $\boldsymbol{d}_{2}\left(f^{p}, h^{p}\right)<1$. Thus we obtain

$$
\left|\bar{\Phi}_{n}(f)-\bar{\Phi}_{n}(h)\right|=\frac{1}{p}\left|\bar{\Phi}_{n}\left(f^{p}\right)-\bar{\Phi}_{n}\left(h^{p}\right)\right| \leq \frac{D_{\Phi_{n}}+\left|\bar{\Phi}_{n}\left(f^{p} h^{-p}\right)\right|}{p} .
$$


It follows from Corollary 3.6 that

$$
\left|\Phi_{n}\left(f^{p} h^{-p}\right)\right| \leq C_{3} d_{2}\left(\mathrm{Id}, f^{p} h^{-p}\right)=C_{3} \boldsymbol{d}_{2}\left(f^{p}, h^{p}\right)<C_{3} .
$$

Thus

$$
\left|\bar{\Phi}_{n}(f)-\bar{\Phi}_{n}(h)\right|<\frac{D_{\bar{\Phi}_{n}}+C_{3}}{p}<\varepsilon .
$$

\section{C Proof of Theorem 2.3}

Let $n \geq 3$ and denote by

$$
\boldsymbol{A}_{n}:=\left\langle\eta_{i, n} \mid 2 \leq i \leq n\right\rangle
$$

the abelian subgroup of $\boldsymbol{P}_{n}$ generated by braids $\eta_{i, n}$ shown in Figure 1. Let $Q\left(\boldsymbol{B}_{n}, \boldsymbol{A}_{n}\right)$ be the space of homogeneous quasimorphisms on $\boldsymbol{B}_{n}$ which are identically zero on the group $\boldsymbol{A}_{n}$. It follows from [4, Theorem 12] that the space $Q\left(\boldsymbol{B}_{n}\right)$ is infinitedimensional. The restriction of every homogeneous quasimorphism on an abelian group is a homomorphism, hence the space $Q\left(\boldsymbol{B}_{n}, \boldsymbol{A}_{n}\right)$ is also infinite-dimensional. The following theorem was proved by Ishida; see [16, Theorem 1.2].

Theorem 3.9 The map $\mathcal{G}_{n}: Q\left(\boldsymbol{B}_{n}\right) \rightarrow Q(\boldsymbol{G})$ is injective.

In particular the map $\mathcal{G}_{n}: Q\left(\boldsymbol{B}_{n}, \boldsymbol{A}_{n}\right) \rightarrow Q(\boldsymbol{G})$ is also injective. It follows from [20, Theorem 2.7] that Morse-type Hamiltonians form a $C^{1}$-dense subset of $\mathcal{H}$, hence Theorem 3.2 and Theorem 3.4 imply that the image of $Q\left(\boldsymbol{B}_{n}, \boldsymbol{A}_{n}\right)$ under the map $\mathcal{G}_{n}$ lies in $Q(\boldsymbol{G}$, Aut $)$ and the proof follows.

\section{D Proof of Theorem 1}

Let us start with the following basic result, which is interesting in its own right.

Lemma 3.10 Let $\Gamma$ be a group and let $V \subset Q(\Gamma)$ be a $k$-dimensional subspace, where $k \in N$. There exist elements $g_{1}, \ldots, g_{k} \in \Gamma$ and $\psi_{1}, \ldots, \psi_{k} \in V$ such that

$$
\psi_{i}\left(g_{j}\right)=\delta_{i j},
$$

where $\delta_{i j}$ is the Kronecker delta.

Proof We prove the statement by induction on the dimension. For $k=1$ it is clearly true. Let $V \subset Q(\Gamma)$ be a $k$-dimensional subspace. According to the induction hypothesis there exist $\varphi_{i} \in V$ and $g_{j} \in \Gamma$ such that $\varphi_{i}\left(g_{j}\right)=\delta_{i j}$, where $1 \leq i, j \leq k-1$. 
Let $\varphi_{k} \in V$ be such that the vectors $\varphi_{1}, \ldots, \varphi_{k}$ are linearly independent. Denote $\psi_{k}:=\varphi_{k}-\sum_{i=1}^{k-1} \varphi_{k}\left(g_{i}\right) \varphi_{i}$. We get that $\psi_{k}\left(g_{j}\right)=0$ for $j=1, \ldots, k-1$. The above linear independence implies (possibly after multiplying $\psi_{k}$ by a nonzero constant) that there exists $g_{k} \in \Gamma$ such that $\psi_{k}\left(g_{k}\right)=1$.

Let $\psi_{i}=\varphi_{i}-\varphi_{i}\left(g_{k}\right) \psi_{k}$, where $1 \leq i \leq k-1$. We obtain that $\psi_{i}\left(g_{k}\right)=0$ and $\psi_{i}\left(g_{j}\right)=\delta_{i j}$ for each $1 \leq i, j \leq k-1$. Thus the $k$-tuples $\psi_{1}, \ldots, \psi_{k}$ and $g_{1}, \ldots, g_{k}$ satisfy the statement of the lemma.

Now we start proving the theorem. Let $r=1 / k$. Denote by $\boldsymbol{D}_{r}$ an open disc in the Euclidean plane of radius $r$ centered at zero. Let

$$
\boldsymbol{G}_{r}:=\operatorname{Diff}\left(\boldsymbol{D}_{r}, \text { area }\right)
$$

be the group of smooth compactly supported area-preserving diffeomorphisms of $\boldsymbol{D}_{r}$. Gambaudo-Ghys construction is valid in the case of $G_{r}$ as well, ie every homogeneous quasimorphism $\varphi_{n}: \boldsymbol{B}_{n} \rightarrow \boldsymbol{R}$ defines a homogeneous quasimorphism $\bar{\Phi}_{n, r}: \boldsymbol{G}_{r} \rightarrow \boldsymbol{R}$. This construction defines a homomorphism $\mathcal{G}_{n, r}: Q\left(\boldsymbol{B}_{n}\right) \rightarrow Q\left(\boldsymbol{G}_{r}\right)$.

The vector space

$$
\operatorname{Im}\left(\left.\mathcal{G}_{n, r}\right|_{Q\left(\boldsymbol{B}_{n}, \boldsymbol{A}_{n}\right)}\right) \subset Q\left(\boldsymbol{G}_{r}, \text { Aut }\right)
$$

is infinite-dimensional for $n \geq 3$. The proof of this fact is identical to the proof of Theorem 2.3. As an immediate consequence of Lemma 3.10 we have the following fact: for each $n \geq 3$ there exist $\left\{g_{i, n}\right\}_{i=1}^{k} \in \boldsymbol{G}_{r}$ and $\left\{\bar{\Phi}_{i, n, r}\right\}_{i=1}^{k} \in \operatorname{Im}\left(\left.\mathcal{G}_{n, r}\right|_{Q\left(\boldsymbol{B}_{n}, \boldsymbol{A}_{n}\right)}\right) \subset$ $Q\left(\boldsymbol{G}_{r}\right.$, Aut $)$ such that

$$
\bar{\Phi}_{i, n, r}\left(g_{j, n}\right)=\delta_{i j},
$$

where $\delta_{i j}$ is the Kronecker delta.

We extend every diffeomorphism in $\boldsymbol{G}_{r}$ by identity on the unit disc $\boldsymbol{D}^{2}$ and get an injective homomorphism $i_{r}: \boldsymbol{G}_{r} \rightarrow \boldsymbol{G}$.

Lemma 3.11 The following identity holds on the space $Q\left(\boldsymbol{B}_{3}, \boldsymbol{A}_{3}\right)$ :

$$
\mathcal{G}_{3, r}=Q\left(i_{r}\right) \circ \mathcal{G}_{3} .
$$

Equivalently, for each $\bar{\Phi}_{3, r} \in \operatorname{Im}\left(\left.\mathcal{G}_{3, r}\right|_{Q\left(\boldsymbol{B}_{3}, \boldsymbol{A}_{3}\right)}\right) \subset Q(\boldsymbol{G}$, Aut $)$ and $g \in \boldsymbol{G}_{r}$ we have

$$
\bar{\Phi}_{3, r}(g)=\bar{\Phi}_{3}\left(i_{r}(g)\right),
$$

where $\bar{\Phi}_{3}$ is defined using the same quasimorphism $\varphi_{3}$ in $Q\left(\boldsymbol{B}_{3}, \boldsymbol{A}_{3}\right)$. 
Proof Denote by $\mathrm{X}_{3}\left(\boldsymbol{D}_{r}\right)$ the space of all ordered 3-tuples of distinct points in $\boldsymbol{D}_{\boldsymbol{r}}$. It follows that

$$
\begin{aligned}
\bar{\Phi}_{3}\left(i_{r}(g)\right) & =\lim _{p \rightarrow+\infty}\left(\int_{\mathbf{X}_{3}\left(\boldsymbol{D}_{r}\right)} \frac{\varphi_{3}\left(\gamma\left(g^{p} ; x\right)\right)}{p} d x+\int_{\mathbf{X}_{3}\left(\boldsymbol{D}^{2}\right) \backslash \mathrm{X}_{3}\left(\boldsymbol{D}_{r}\right)} \frac{\varphi_{3}\left(\gamma\left(g^{p} ; x\right)\right)}{p} d x\right) \\
& =\bar{\Phi}_{3, r}(g)+\int_{\mathbf{X}_{3}\left(\boldsymbol{D}^{2}\right) \backslash \mathrm{X}_{3}\left(\boldsymbol{D}_{r}\right)} \lim _{p \rightarrow+\infty} \frac{\varphi_{3}\left(\gamma\left(g^{p} ; x\right)\right)}{p} d x .
\end{aligned}
$$

By definition, $i_{r}(g)=\mathrm{Id}$ on $\boldsymbol{D}^{2} \backslash \boldsymbol{D}_{r}$. It follows that for $x \in \mathrm{X}_{3}\left(\boldsymbol{D}^{2}\right) \backslash \mathrm{X}_{3}\left(\boldsymbol{D}_{r}\right)$ the braid

$$
\gamma\left(g^{p} ; x\right)=\alpha_{1, p, x} \circ \beta_{p, x} \circ \eta_{2,3}^{m_{x, p}} \circ \beta_{p, x}^{-1} \circ \alpha_{2, p, x},
$$

where the length of the braids $\alpha_{1, p, x}$ and $\alpha_{2, p, x}$ is bounded for all $p$ and $x$. It follows that for all $x \in \mathrm{X}_{3}\left(\boldsymbol{D}^{2}\right) \backslash \mathrm{X}_{3}\left(\boldsymbol{D}_{r}\right)$ we have

$$
\lim _{p \rightarrow+\infty} \frac{\varphi_{3}\left(\gamma\left(g^{p} ; x\right)\right)}{p}=\lim _{p \rightarrow+\infty} \frac{\left.\varphi_{3}\left(\beta_{p, x} \circ \eta_{2,3}^{m_{x, p}} \circ \beta_{p, x}^{-1}\right)\right)}{p}=0,
$$

where the last equality follows from the fact that homogeneous quasimorphisms are invariant under conjugation and that $\varphi_{3}\left(\eta_{2,3}\right)=0$, because $\varphi_{3} \in Q\left(\boldsymbol{B}_{3}, \boldsymbol{A}_{3}\right)$. Hence

$$
\int_{\mathbf{X}_{3}\left(\boldsymbol{D}^{2}\right) \backslash \mathrm{X}_{3}\left(\boldsymbol{D}_{r}\right)} \lim _{p \rightarrow+\infty} \frac{\varphi_{3}\left(\gamma\left(g^{p} ; x\right)\right)}{p} d x=0,
$$

and the proof of the lemma follows.

Remark 3.12 Let inc: $\boldsymbol{B}_{n-1} \rightarrow \boldsymbol{B}_{n}$ be the standard inclusion of braid groups. A homogeneous quasimorphism $\varphi_{n} \in Q\left(\boldsymbol{B}_{n}\right)$ is called kernel quasimorphism if $\varphi_{n}(\alpha)=0$ for each $\alpha \in \operatorname{Im}($ inc). In the proof of Lemma 3.11 we used the fact that the space of kernel quasimorphisms on $\boldsymbol{B}_{3}$ contains the space $Q\left(\boldsymbol{B}_{3}, \boldsymbol{A}_{3}\right)$, ie we used the fact that $\varphi_{3}\left(\eta_{2,3}\right)=0$. If we replace the space $Q\left(\boldsymbol{B}_{n}, \boldsymbol{A}_{n}\right)$ by the space of kernel quasimorphisms on $\boldsymbol{B}_{n}$, then Lemma 3.11 will hold for $n>3$. In what follows we use the fact that the space $Q\left(\boldsymbol{B}_{3}, \boldsymbol{A}_{3}\right)$ is infinite-dimensional, and it is not known what is the dimension of the space of kernel quasimorphisms on $\boldsymbol{B}_{n}$ for $n>3$ (for more information about kernel quasimorphisms, see [18]), hence we restrict ourselves to the case $n=3$.

Let us proceed with the proof of Theorem 1. For $1 \leq j \leq k$, denote $g_{j}:=i_{r}\left(g_{j, 3}\right) \in \boldsymbol{G}$. It follows from Lemma 3.11 that

$$
\bar{\Phi}_{i, 3}\left(g_{j}\right)=\delta_{i j}
$$

where $\bar{\Phi}_{i, 3} \in Q(\boldsymbol{G}$, Aut $)$ is defined using the same quasimorphism in $Q\left(\boldsymbol{B}_{3}, \boldsymbol{A}_{3}\right)$ as $\bar{\Phi}_{i, 3, r} \in Q\left(\boldsymbol{G}_{r}\right.$, Aut $)$. Recall that the support of each $g_{j}$ is contained inside the disc 
$\boldsymbol{D}_{r}$. Since $r=1 / k$, there exists a family of diffeomorphisms $\left\{h_{j}\right\}_{j=1}^{k}$ in $\boldsymbol{G}$, such that $h_{j} \circ g_{j} \circ h_{j}^{-1}$ and $h_{i} \circ g_{i} \circ h_{i}^{-1}$ have disjoint supports for all different $i$ and $j$ between 1 and $k$. It follows from the definition of Calabi homomorphism, see Remark 2.2, that there exists a family $\left\{g_{i}^{\prime}\right\}_{i=1}^{k}$ of autonomous diffeomorphisms in $\boldsymbol{G}$ such that:

- The diffeomorphisms $g_{i}^{\prime}$ and $g_{j}^{\prime}$ have disjoint supports for $i \neq j$, and the diffeomorphisms $g_{i}^{\prime}$ and $h_{j} \circ g_{j} \circ h_{j}^{-1}$ have disjoint supports for all $1 \leq i, j \leq k$.

- For each $1 \leq i \leq k$ we have $\mathcal{C}\left(g_{i}^{\prime}\right)=\mathcal{C}\left(h_{i} \circ g_{i} \circ h_{i}^{-1}\right)$.

Denote $f_{i}:=h_{i} \circ g_{i} \circ h_{i}^{-1} \circ\left(g_{i}^{\prime}\right)^{-1}$ and let $\boldsymbol{K}:=\operatorname{ker} \mathcal{C}$. Note that all of the $f_{i}$ have disjoint supports, $\mathcal{C}\left(f_{i}\right)=0$, each $f_{i}$ lies in $\boldsymbol{K}$ and they generate a free abelian group of rank $k$. Let

$$
\Psi: Z^{k} \longrightarrow \boldsymbol{G}
$$

where $\Psi\left(d_{1}, \ldots, d_{k}\right)=f_{1}^{d_{1}} \circ \cdots \circ f_{k}^{d_{k}}$. It is obvious that $\Psi$ is a monomorphism whose image lies in $\boldsymbol{K}$. In order to complete the proof of the theorem it is left to show that $\Psi$ is a bi-Lipschitz map, ie we are going to show that there exists a constant $A \geq 1$ such that

$$
A^{-1} \sum_{i=1}^{k}\left|d_{i}\right| \leq\left\|f_{1}^{d_{1}} \circ \cdots \circ f_{k}^{d_{k}}\right\|_{\text {Aut }} \leq A \sum_{i=1}^{k}\left|d_{i}\right| .
$$

We have the following equalities:

$$
\bar{\Phi}_{i, 3}\left(f_{j}\right)=\bar{\Phi}_{i, 3}\left(h_{j} \circ g_{j} \circ h_{j}^{-1} \circ\left(g_{j}^{\prime}\right)^{-1}\right)=\bar{\Phi}_{i, 3}\left(g_{j}\right)+\bar{\Phi}_{i, 3}\left(\left(g_{j}^{\prime}\right)^{-1}\right)=\bar{\Phi}_{i, 3}\left(g_{j}\right)=\delta_{i j} .
$$

The second equality follows from the fact that every homogeneous quasimorphism is invariant under conjugation and it behaves as a homomorphism on every pair of commuting elements. The third equality follows from the fact that $\left(g_{j}^{\prime}\right)^{-1}$ is an autonomous diffeomorphism and $\bar{\Phi}_{i, 3} \in Q(\boldsymbol{G}$, Aut $)$, and the forth equality is (4). Since all $f_{i}$ commute with each other and $\bar{\Phi}_{i, 3}\left(f_{j}\right)=\delta_{i j}$, we obtain

$$
\left\|f_{1}^{d_{1}} \circ \cdots \circ f_{k}^{d_{k}}\right\|_{\text {Aut }} \geq \frac{\left|\bar{\Phi}_{i, 3}\left(f_{1}^{d_{1}} \circ \cdots \circ f_{k}^{d_{k}}\right)\right|}{D_{\bar{\Phi}_{i, 3}}}=\frac{\left|d_{i}\right|}{D_{\bar{\Phi}_{i, 3}}},
$$

where $D \bar{\Phi}_{i, 3}$ is the defect of the quasimorphism $\bar{\Phi}_{i, 3}$. The defect $D \bar{\Phi}_{i, 3} \neq 0$, because each $\bar{\Phi}_{i, 3} \in Q(\boldsymbol{G}$, Aut $)$ and hence it is not a homomorphism. We set $\mathfrak{D}_{k}:=\max _{i} D_{\Phi_{i, 3}}$ and obtain the following inequality:

$$
\left\|f_{1}^{d_{1}} \circ \cdots \circ f_{k}^{d_{k}}\right\|_{\text {Aut }} \geq\left(k \cdot \mathfrak{D}_{k}\right)^{-1} \sum_{i=1}^{k}\left|d_{i}\right| .
$$


Denote $\mathfrak{M}_{f}:=\max _{i}\left\|f_{i}\right\|_{\text {Aut }}$. Now we have the following inequality:

$$
\left\|f_{1}^{d_{1}} \circ \cdots \circ f_{k}^{d_{k}}\right\|_{\text {Aut }} \leq \sum_{i=1}^{k}\left|d_{i}\right| \cdot\left\|f_{i}\right\|_{\text {Aut }} \leq \mathfrak{M}_{f} \cdot \sum_{i=1}^{k}\left|d_{i}\right| .
$$

Inequalities (5) and (6) conclude the proof of the theorem.

Remark 3.13 In fact, the proof of Theorem 1 shows that for each $k \in N$ there exists a bi-Lipschitz embedding of $\boldsymbol{Z}^{k}$ into $\left(\boldsymbol{G}, \boldsymbol{d}_{\text {Aut }}\right)$ with the image contained in a $C^{0}$-neighborhood of the identity diffeomorphism in $\boldsymbol{G}$.

\section{A relation between $Q(G)$ and $Q\left(F_{2}\right)$}

Let $\boldsymbol{F}_{2}$ denote the free group on two generators, and let $\sigma_{1}$ and $\sigma_{2}$ denote the Artin generators of $\boldsymbol{B}_{3}$. The center of both $\boldsymbol{B}_{3}$ and $\boldsymbol{P}_{3}$ is a cyclic group generated by an element $\Delta=\eta_{2,3} \cdot \eta_{3,3}$. One can show that $\boldsymbol{P}_{3}$ is generated by $\sigma_{1}^{2}, \sigma_{2}^{2}, \Delta$ and $\boldsymbol{P}_{3} \cong \boldsymbol{F}_{2} \times Z\left(\boldsymbol{P}_{3}\right)$, where $\boldsymbol{F}_{2}=\left\langle\sigma_{1}^{2}, \sigma_{2}^{2}\right\rangle$. In what follows we describe a monomorphism from $\boldsymbol{F}_{2}$ to $\boldsymbol{G}$ and study the induced map from $Q(\boldsymbol{G})$ to $Q\left(\boldsymbol{F}_{2}\right)$, which is infinitedimensional by the theorem of Brooks [8].

Let $U_{1}, U_{2}, U_{3} \subset D^{2}$ be open subsets each diffeomorphic to a disc, such that $\operatorname{area}\left(U_{i}\right) \geq \pi / 4$. We also require that $z_{i} \in U_{i}$, where $z=\left(z_{1}, z_{2}, z_{3}\right)$ is a basepoint for $\pi_{1}\left(\mathrm{X}_{3}\left(\boldsymbol{D}^{2}\right)\right) \cong \boldsymbol{P}_{3}$. For pairs $\left(U_{1}, U_{2}\right)$ and $\left(U_{2}, U_{3}\right)$ let $W_{12} \subset V_{12}$ and $W_{23} \subset V_{23}$ be pairs of two open subsets of $\boldsymbol{D}^{2}$, each diffeomorphic to a disc, such that $U_{1} \cup U_{2} \subset W_{12}$, $U_{2} \cup U_{3} \subset W_{23}, V_{12} \cap U_{3}=\varnothing$ and $V_{23} \cap U_{1}=\varnothing$. Let $\left\{h_{t}\right\}$ be a path in $\boldsymbol{G}$ which rotates $W_{12}$ once, and is identity on the outside of $V_{12}$ and on a small neighborhood of $\partial V_{12}$. Similarly, let $\left\{h_{t}^{\prime}\right\}$ be a path in $\boldsymbol{G}$ which rotates $W_{23}$ once, and is identity on the outside of $V_{23}$ and on a small neighborhood of $\partial V_{23}$.

Let $U:=U_{1} \cup U_{2} \cup U_{3}$ and let $\boldsymbol{G}_{U}$ be the subgroup of $\boldsymbol{G}$ which consists of diffeomorphisms that preserve pointwise the set $U$. Let

$$
\operatorname{Tr}: \boldsymbol{G}_{U} \longrightarrow \boldsymbol{P}_{3}
$$

where $\operatorname{Tr}(g)$ is the homotopy class of the loop $\left(g_{t}\left(z_{1}\right), g_{t}\left(z_{2}\right), g_{t}\left(z_{3}\right)\right)$ in $\mathrm{X}_{3}\left(\boldsymbol{D}^{2}\right)$. Here $\left\{g_{t}\right\}_{t=0}^{1}$ is any isotopy from the identity map to $g$. Since the map $\operatorname{Tr}$ is a homomorphism, which sends $h_{1}$ to $\sigma_{1}^{2}$ and $h_{1}^{\prime}$ to $\sigma_{2}^{2}$, the diffeomorphisms $h_{1}$ and $h_{1}^{\prime}$ generate a free group in $\boldsymbol{G}$. Let

$$
s_{U}: \boldsymbol{F}_{2} \longrightarrow \boldsymbol{G}
$$

be a monomorphism, where $s_{U}\left(\sigma_{1}^{2}\right)=h_{1}$ and $s_{U}\left(\sigma_{2}^{2}\right)=h_{1}^{\prime}$. Denote $a_{i}=\operatorname{area}\left(U_{i}\right)$ and $a=\operatorname{area}(U)$. 
Theorem 4.1 Let $Q\left(s_{U}\right): Q(\boldsymbol{G}$, Aut $) \rightarrow Q\left(\boldsymbol{F}_{2}\right)$ be the map induced by the homomorphism $s_{U}$. Then

$$
\lim _{a \rightarrow \pi} \operatorname{dim}\left(\operatorname{Im}\left(Q\left(s_{U}\right)\right)\right)=\infty
$$

Proof Let $N \in \boldsymbol{N}$. We are going to show that there exists $\varepsilon>0$ such that whenever $|a-\pi|<\varepsilon$ we have $\operatorname{dim}\left(\operatorname{Im}\left(Q\left(s_{U}\right)\right)\right) \geq N$. Notice that every $\varphi \in Q\left(\boldsymbol{B}_{3}, \boldsymbol{A}_{3}\right)$ vanishes on $\Delta$. Since $\operatorname{dim}\left(Q\left(\boldsymbol{B}_{3}, \boldsymbol{A}_{3}\right)\right)=\infty$ and $\boldsymbol{P}_{3}$ is a subgroup of finite index in $\boldsymbol{B}_{3}$, it follows from Lemma 3.10 that there exists a family of quasimorphisms $\left\{\varphi_{i}\right\}_{i=1}^{N}$ in $Q\left(\boldsymbol{B}_{3}, \boldsymbol{A}_{3}\right)$ and a family of braids $\left\{\beta_{i}\right\}_{i=1}^{N}$ which are words in $\sigma_{1}^{2}, \sigma_{2}^{2}$, such that $\varphi_{i}\left(\beta_{j}\right)=\delta_{i j}$. Denote $g_{U, i}:=s_{U}\left(\beta_{i}\right)$, ie each $g_{U, i}$ is a time-one map of an isotopy $g_{t, i}$ which is a composition of a number of isotopies $h_{t}$ and $h_{t}^{\prime}$ that twist the $U_{j}$ in the form of the braid $\beta_{i}$. We are going to show that there exists $\varepsilon>0$, such that if $|a-\pi|<\varepsilon$ then the matrix

$$
M_{N \times N}:=\left(\mathcal{G}_{3}\left(\varphi_{i}\right)\left(g_{U, j}\right)\right)_{1 \leq i \leq j \leq N}
$$

is nonsingular, where $\mathcal{G}_{3}: Q\left(\boldsymbol{B}_{3}, \boldsymbol{A}_{3}\right) \rightarrow Q(\boldsymbol{G}$, Aut $)$. This will imply that the vectors $\left\{Q\left(s_{U}\right)\left(\mathcal{G}_{3}\left(\varphi_{i}\right)\right)\right\}_{i=1}^{N}$ are linearly independent in $Q\left(\boldsymbol{F}_{2}\right)$.

It is easy to show that there exists $\varepsilon^{\prime}>0$, such that each $N \times N$ matrix with entries $m_{i j}$ is nonsingular provided that $1<m_{i i}<12$ and $\left|m_{i j}\right|<\varepsilon^{\prime}$ for all $i \neq j$. Denote $\mathrm{X}_{3}(U):=\mathrm{X}_{3}\left(\bigcup_{i=1}^{3} U_{i}\right)$. Since each $\varphi_{i} \in Q\left(\boldsymbol{B}_{3}, \boldsymbol{A}_{3}\right)$ is invariant under conjugation in $\boldsymbol{B}_{3}$ and vanishes on the braid $\eta_{3,3}$ we have

$$
\int_{\mathrm{X}_{3}(U)} \lim _{p \rightarrow+\infty} \frac{\varphi_{i}\left(\gamma\left(g_{U, j}^{p} ; x\right)\right)}{p} d x= \begin{cases}0 & \text { if } i \neq j, \\ 6 a_{1} \cdot a_{2} \cdot a_{3} & \text { if } i=j .\end{cases}
$$

It follows that

$$
\begin{aligned}
\mathcal{G}_{3}\left(\varphi_{i}\right)\left(g_{U, j}\right) & \begin{cases}\int_{X_{3}\left(\boldsymbol{D}^{2}\right) \backslash X_{3}(U)} \lim _{p \rightarrow+\infty} \frac{\varphi_{i}\left(\gamma\left(g_{U, j}^{p} ; x\right)\right)}{p} d x & \text { if } i \neq j, \\
6 a_{1} \cdot a_{2} \cdot a_{3}+\int_{X_{3}\left(\boldsymbol{D}^{2}\right) \backslash X_{3}(U)} \lim _{p \rightarrow+\infty} \frac{\varphi_{i}\left(\gamma\left(g_{U, i}^{p} ; x\right)\right)}{p} d x & \text { otherwise. }\end{cases}
\end{aligned}
$$

For $x \in \mathrm{X}_{3}\left(\boldsymbol{D}^{2}\right)$ denote by $\operatorname{cr}\left(g_{U, i}^{p} ; x\right)$ the length of the word in generators $\sigma_{1}, \sigma_{2}$, which represents the braid $\gamma\left(g_{U, i}^{p} ; x\right)$ and is given by $p$ concatenations of flows $g_{t, i}$. Let

$$
\operatorname{cr}\left(\beta_{i}\right):=\operatorname{cr}\left(g_{U, i} ; z\right) \quad \text { and } \quad \mathfrak{M}_{c r}:=\max _{1 \leq i \leq N} \operatorname{cr}\left(\beta_{i}\right)
$$


where $z=\left(z_{1}, z_{2}, z_{3}\right)$. It follows from the construction of diffeomorphisms $g_{U, i}$, that for each $x \in \mathrm{X}_{3}\left(\boldsymbol{D}^{2}\right)$ and $1 \leq i \leq N$ we have

$$
\lim _{p \rightarrow+\infty} \frac{c r\left(\gamma\left(g_{U, i}^{p} ; x\right)\right)}{p} \leq \mathfrak{M}_{c r}
$$

For each $\gamma \in \boldsymbol{B}_{3}$ denote by $l(\gamma)$ the word length of $\gamma$ with respect to the generating set $\sigma_{1}, \sigma_{2}$. Since each $\varphi_{i}$ is a homogenous quasimorphism that vanishes on $\sigma_{1}, \sigma_{2}$, we obtain

$$
\left|\varphi_{i}(\gamma)\right| \leq D_{\varphi_{i}} \cdot l(\gamma)
$$

Denote

$$
\mathfrak{M}_{D}:=\max _{1 \leq i \leq N} D_{\varphi_{i}}
$$

It follows that for each $x \in \mathrm{X}_{3}\left(\boldsymbol{D}^{2}\right)$ and $1 \leq i, j \leq N$ we have

$$
\begin{aligned}
\lim _{p \rightarrow+\infty} \frac{\left|\varphi_{i}\left(\gamma\left(g_{U, j}^{p} ; x\right)\right)\right|}{p} & \leq \mathfrak{M}_{D} \lim _{p \rightarrow+\infty} \frac{\left|l\left(\gamma\left(g_{U, j}^{p} ; x\right)\right)\right|}{p} \\
& \leq \mathfrak{M}_{D} \lim _{p \rightarrow+\infty} \frac{\operatorname{cr}\left(\gamma\left(g_{U, j}^{p} ; x\right)\right)}{p} \leq \mathfrak{M}_{D} \cdot \mathfrak{M}_{c r} .
\end{aligned}
$$

Take $\varepsilon>0$, such that

$$
\mathfrak{M}_{D} \cdot \mathfrak{M}_{c r} \cdot \operatorname{area}\left(\mathrm{X}_{3}\left(D^{2}\right) \backslash \mathrm{X}_{3}(U)\right)<\min \left\{\frac{1}{10}, \varepsilon^{\prime}\right\} \text {. }
$$

Equality (7) yields

$$
\left|\mathcal{G}_{3}\left(\varphi_{i}\right)\left(g_{U, j}\right)\right| \leq \varepsilon^{\prime} \quad \text { if } i \neq j \quad \text { and } \quad 1 \leq \mathcal{G}_{3}\left(\varphi_{i}\right)\left(g_{U, i}\right) \leq 12
$$

hence the matrix $M_{N \times N}$ is nonsingular and the proof follows.

\section{Comparison of bi-invariant metrics on $G$ and other com- ments}

\section{A The Hofer metric}

The most famous metric on the group of Hamiltonian diffeomorphisms of a symplectic manifold $(M, \omega)$ is the Hofer metric; see Hofer [15] and Lalonde and McDuff [17]. The associated norm is defined by

$$
\|f\|_{\text {Hofer }}:=\inf _{F_{t}} \int_{0}^{1} \operatorname{osc}\left(F_{t}\right) d t
$$


where $F_{t}$ is a compactly supported Hamiltonian function generating the Hamiltonian flow $f_{t}$ from the identity to $f=f_{1}$. The oscillation norm is defined by

$$
\operatorname{osc}(F)=\max _{M} F-\min _{M} F .
$$

Example 5.1 Let $f \in \boldsymbol{G}$ be a diffeomorphism generated by a time independent and nonnegative Hamiltonian function $F$. This implies that all powers of $f$ are also autonomous and hence $\left\|f^{n}\right\|_{\text {Aut }}=1$ for all $n \in Z$. On the other hand, the Calabi homomorphism is positive on $f$ and hence $\left\|f^{n}\right\|_{\text {Hofer }} \geq$ const $|n| \mathcal{C}(f)$, for some positive constant.

Also, $\left\|f^{1 / n}\right\|_{\text {Aut }}=1$ but $\lim _{n \rightarrow \infty}\left\|f^{1 / n}\right\|_{\text {Hofer }}=0$. Here $f^{1 / n}$ is the unique diffeomorphism in the flow generated by $F$ such that its $n$-th power is equal to $f$. This shows that the identity homomorphism between the autonomous metric and the Hofer metric is not Lipschitz in neither direction.

\section{B The restricted autonomous metric}

Let $S_{r} \subset \operatorname{Ham}(M, \omega)$ be the set of autonomous diffeomorphisms generated by Hamiltonian functions with the $L^{\infty}$-norm bounded by $r>0$. This set is invariant under conjugations and hence the corresponding word metric is bi-invariant. We call it the restricted autonomous metric and denote the corresponding norm by $\|f\|_{r}$. For all $r$ these metrics are Lipschitz equivalent. Indeed, it is easy to check that if $r \leq R$ then

$$
\|f\|_{R} \leq\|f\|_{r} \leq\lceil R / r\rceil\|f\|_{R}
$$

for all $f \in \operatorname{Ham}(M, \omega)$. Moreover, we have that $\|f\|_{\text {Aut }} \leq\|f\|_{r}$ for every $r$. This trivially implies that the main results of the paper hold for the restricted autonomous metric.

Let $f \in \boldsymbol{G}$ be such that $f=h_{1} \circ \cdots \circ h_{k}$ with each $h_{i}$ is autonomous generated by a Hamiltonian $H_{i}$ of the oscillation norm smaller than $r$. We have

$$
\begin{aligned}
\mathcal{C}(f)=\int_{0}^{1} d t \int_{\boldsymbol{D}^{2}} F_{t} \omega & =\int_{0}^{1} d t \int_{\boldsymbol{D}^{2}} \sum_{i=1}^{k} H_{i}\left(\left(h_{1, t} \circ \cdots \circ h_{i-1, t}\right)^{-1}\right) \omega \\
& \leq \sum_{i=1}^{k} \text { osc } H_{i} \leq k r .
\end{aligned}
$$

We thus obtain the following estimate:

$$
\mathcal{C}(f) / r \leq\|f\|_{r}
$$

which proves that the restricted autonomous norm is not equivalent to the autonomous norm for there are autonomous diffeomorphisms with arbitrarily big Calabi invariant. 


\section{C Fragmentation metrics}

Let $U \subset M$ be a set with nonempty interior. The fragmentation metric $\boldsymbol{d}_{U}$ is a word metric defined with respect to the generating set consisting of diffeomorphisms conjugated to ones supported in $U$. Such a set is invariant under conjugations by construction and hence the fragmentation metric is bi-invariant.

It follows from the proof of Theorem 1 that there is a diffeomorphism $f$ supported in the set $U$ such that $f$ has arbitrarily big autonomous norm. Clearly, the fragmentation norm of $f$ is equal to one.

Example 5.2 Suppose that $U \subset D^{2}$ is a disc of radius $1 / 2$. According to Biran, Entov and Polterovich [5], the space of homogeneous Calabi quasimorphisms on $\operatorname{Ham}\left(\boldsymbol{D}^{2}\right)$ is infinite-dimensional. The Calabi property means that the restriction of a quasimorphism to the subgroup of diffeomorphisms supported on a displaceable subset is equal to the Calabi homomorphism.

Consider the subgroup $\boldsymbol{K}=\operatorname{ker} \mathcal{C} \subset \boldsymbol{G}$. It is generated up to conjugation by diffeomorphisms supported in $U$ and hence the fragmentation metric is defined on $\boldsymbol{K}$. (In the next section we explain that this metric is equal to the autonomous metric induced from $\boldsymbol{G}$.) Let $q: \boldsymbol{K} \rightarrow \boldsymbol{R}$ be a Calabi quasimorphism. Since it is trivial on the generators it is Lipschitz with respect to the fragmentation norm.

It follows from the proof of [5, Theorem 2.3] that there is an autonomous diffeomorphism $f \in \boldsymbol{K}$ and a homogeneous Calabi quasimorphism $q: \boldsymbol{K} \rightarrow \boldsymbol{R}$ such that $q(f)>0$. This implies that $f^{n}$ can have arbitrarily big fragmentation norm. Its autonomous norm is equal to one.

\section{D The kernel of the Calabi homomorphism}

This is a remark on the geometry of the inclusion $\operatorname{ker} \mathcal{C}=\boldsymbol{K} \rightarrow \boldsymbol{G}$ with respect to the autonomous metric. Observe that the kernel of the Calabi homomorphism is generated by autonomous diffeomorphisms and let $\|g\|_{\text {Aut }^{\prime}}$ denotes the corresponding autonomous norm of $g \in \boldsymbol{K}$.

Lemma 5.3 Let $\boldsymbol{i}: \boldsymbol{K} \rightarrow \boldsymbol{G}$ be the inclusion. Then

$$
\|g\|_{\text {Aut }}=\|\boldsymbol{i}(g)\|_{\text {Aut }}
$$

for every $g \in \boldsymbol{K}$. 
Proof By definition for each $g \in \boldsymbol{K}$ we have $\|\boldsymbol{i}(g)\|_{\text {Aut }} \leq\|g\|_{\text {Aut }^{\prime}}$. Let $g \neq$ Id and suppose that $\|\boldsymbol{i}(g)\|_{\text {Aut }}=m$. It means that the diffeomorphism $g=h_{1} \circ \cdots \circ h_{m}$ for some autonomous diffeomorphisms $h_{i}$. It is straightforward to construct autonomous diffeomorphisms $f_{1}, \ldots, f_{m-1}$ such that:

- The diffeomorphisms $f_{i}$ and $f_{j}$ have disjoint supports for $i \neq j$, and the diffeomorphisms $f_{i}$ and $h_{j}$ have disjoint supports for all $1 \leq i \leq m-1$ and $1 \leq j \leq m$.

- $\mathcal{C}\left(f_{1}\right)=\mathcal{C}\left(h_{1}\right)$ and $\mathcal{C}\left(f_{i}\right)=\mathcal{C}\left(f_{i-1} \circ h_{i}\right)$ for $2 \leq i \leq m-1$.

For example, we can take autonomous diffeomorphisms $f_{i}$ disjointly supported away from the union of the supports of the $h_{j}$ and with appropriate values of the Calabi homomorphism. We can write $g$ as follows:

$$
g=\left(h_{1} \circ f_{1}^{-1}\right) \circ\left(f_{1} \circ h_{2} \circ f_{2}^{-1}\right) \circ \cdots \circ\left(f_{m-2} \circ h_{m-2} \circ f_{m-1}^{-1}\right) \circ\left(f_{m-1} \circ h_{m}\right) .
$$

Note that

$$
\mathcal{C}\left(h_{1} \circ f_{1}^{-1}\right)=\mathcal{C}\left(f_{i-1} \circ h_{i} \circ f_{i}^{-1}\right)=0
$$

for $2 \leq i \leq m-1$, and $\mathcal{C}\left(f_{m-1} \circ h_{m}\right)=0$ because $\mathcal{C}(g)=0$. Since each $h_{i}$ commutes with each $f_{j}$ and each $f_{i}$ commutes with each $f_{j}$, the diffeomorphisms $h_{1} \circ f_{1}^{-1}$, $f_{i-1} \circ h_{i} \circ f_{i}^{-1}$ for $2 \leq i \leq m-1$, and $f_{m-1} \circ h_{m}$ are autonomous diffeomorphisms, which finishes the proof.

Acknowledgments Both authors would like to thank the anonymous referee for careful reading of our paper and for his/her helpful comments and remarks. This work has been done during the first author stay in Aberdeen and in Mathematisches Forschungsinstitut Oberwolfach. The first author wishes to express his gratitude to both institutes. He was supported by ESF grant number 4824 and by the Oberwolfach Leibniz fellowship. The visit in Aberdeen was supported by the CAST network.

\section{References}

[1] V I Arnold, B A Khesin, Topological methods in hydrodynamics, Applied Mathematical Sciences 125, Springer, New York (1998) MR1612569

[2] A Banyaga, Sur la structure du groupe des difféomorphismes qui préservent une forme symplectique, Comment. Math. Helv. 53 (1978) 174-227 MR490874

[3] M Benaim, J-M Gambaudo, Metric properties of the group of area preserving diffeomorphisms, Trans. Amer. Math. Soc. 353 (2001) 4661-4672 MR1851187

[4] M Bestvina, K Fujiwara, Bounded cohomology of subgroups of mapping class groups, Geom. Topol. 6 (2002) 69-89 MR1914565 
[5] P Biran, M Entov, L Polterovich, Calabi quasimorphisms for the symplectic ball, Commun. Contemp. Math. 6 (2004) 793-802 MR2100764

[6] M Brandenbursky, On quasi-morphisms from knot and braid invariants, J. Knot Theory Ramifications 20 (2011) 1397-1417 MR2851716

[7] M Brandenbursky, Quasi-morphisms and $L^{p}$-metrics on groups of volumepreserving diffeomorphisms, J. Topol. Anal. 4 (2012) 255-270 MR2949242

[8] R Brooks, Some remarks on bounded cohomology, from: "Riemann surfaces and related topics”, (I Kra, B Maskit, editors), Ann. of Math. Stud. 97, Princeton Univ. Press (1981) 53-63 MR624804

[9] D Burago, S Ivanov, L Polterovich, Conjugation-invariant norms on groups of geometric origin, from: "Groups of diffeomorphisms", (R Penner, D Kotschick, T Tsuboi, N Kawazumi, T Kitano, Y Mitsumatsu, editors), Adv. Stud. Pure Math. 52, Math. Soc. Japan, Tokyo (2008) 221-250 MR2509711

[10] E Calabi, On the group of automorphisms of a symplectic manifold, from: "Problems in analysis”, (R C Gunning, editor), Princeton Univ. Press (1970) 1-26 MR0350776

[11] D Calegari, scl, MSJ Memoirs 20, Mathematical Society of Japan, Tokyo (2009) MR2527432

[12] J-M Gambaudo, É Ghys, Enlacements asymptotiques, Topology 36 (1997) 1355-1379 MR1452855

[13] J-M Gambaudo, É Ghys, Commutators and diffeomorphisms of surfaces, Ergodic Theory Dynam. Systems 24 (2004) 1591-1617 MR2104597

[14] J-M Gambaudo, M Lagrange, Topological lower bounds on the distance between area preserving diffeomorphisms, Bol. Soc. Brasil. Mat. 31 (2000) 9-27 MR1754952

[15] H Hofer, On the topological properties of symplectic maps, Proc. Roy. Soc. Edinburgh Sect. A 115 (1990) 25-38 MR1059642

[16] T Ishida, Quasi-morphisms on the group of area-preserving diffeomorphisms of the 2-disk via braid groups, to appear in Proc. Amer. Math. Soc. arXiv:1204.4056

[17] F Lalonde, D McDuff, The geometry of symplectic energy, Ann. of Math. 141 (1995) 349-371 MR1324138

[18] A V Malyutin, Operators in the spaces of pseudocharacters of braid groups, Algebra $\mathrm{i}$ Analiz 21 (2009) 136-165 MR2553045 In Russian; translated in St. Petersburg Math. J. 21 (2010) 261-280

[19] D McDuff, D Salamon, Introduction to symplectic topology, 2nd edition, Oxford University Press (1998) MR1698616

[20] J Milnor, Lectures on the h-cobordism theorem, Princeton Univ. Press (1965) MR0190942 
[21] L Polterovich, The geometry of the group of symplectic diffeomorphisms, Lectures in Mathematics ETH Zürich 12, Birkhäuser, Basel (2001) MR1826128

[22] T Tsuboi, The Calabi invariant and the Euler class, Trans. Amer. Math. Soc. 352 (2000) 515-524 MR1487633

Department of Mathematics, Vanderbilt University 1326 Stevenson Center, Nashville, TN 37240, USA

Institute of Mathematics, University of Aberdeen Aberdeen AB24 3UE, UK

michael.brandenbursky@vanderbilt.edu, kedra@abdn.ac.uk

Received: 20 July 2012 Revised: 18 September 2012 